\title{
Degree Splitting of Root Square Mean Graphs
}

\author{
S. S. Sandhya1, S. Somasundaram ${ }^{2}$, S. Anusa ${ }^{3}$ \\ ${ }^{1}$ Department of Mathematics, Sree Ayyappa College for Women, Chunkankadai, India \\ ${ }^{2}$ Department of Mathematics, Manonmaniam Sundaranar University, Tirunelveli, India \\ ${ }^{3}$ Department of Mathematics, Arunachala College of Engineering for Women, Vellichanthai, India \\ Email: anu12343s@gmail.com
}

Received 15 April 2015; accepted 30 May 2015; published 2 June 2015

Copyright (C) 2015 by authors and Scientific Research Publishing Inc.

This work is licensed under the Creative Commons Attribution International License (CC BY).

http://creativecommons.org/licenses/by/4.0/

c) (i)

\section{Abstract}

Let $f: V(G) \rightarrow\{1,2, \cdots, q+1\}$ be an injective function. For a vertex labeling $f$, the induced edge

labeling $f^{*}(e=u v)$ is defined by, $f^{*}(e=u v)=\left[\sqrt{\frac{f(u)^{2}+f(v)^{2}}{2}}\right\rceil$ or $\left\lfloor\sqrt{\frac{f(u)^{2}+f(v)^{2}}{2}}\right\rfloor$; then, the edge labels are distinct and are from $\{1,2, \cdots, q\}$. Then $f$ is called a root square mean labeling of $G$. In this paper, we prove root square mean labeling of some degree splitting graphs.

\section{Keywords}

Graph, Path, Cycle, Degree Splitting Graphs, Root Square Mean Graphs, Union of Graphs

\section{Introduction}

The graphs considered here are simple, finite and undirected. Let $V(G)$ denote the vertex set and $E(G)$ denote the edge set of $G$. For detailed survey of graph labeling we refer to Gallian [1]. For all other standard terminology and notations we follow Harary [2]. The concept of mean labeling on degree splitting graph was introduced in [3]. Motivated by the authors we study the root square mean labeling on degree splitting graphs. Root square mean labeling was introduced in [4] and the root square mean labeling of some standard graphs was proved in [5]-[11]. The definitions and theorems are useful for our present study.

Definition 1.1: A graph $G=(V, E)$ with $p$ vertices and $q$ edge is called a root square mean graph if it is possible to label the vertices $x \in V$ with distinct labels $f(x)$ from $1,2, \cdots, q+1$ in such a way that when 
each edge $e=u v$ is labeled with $f(e=u v)=\left\lceil\sqrt{\frac{f(u)^{2}+f(v)^{2}}{2}}\right\rceil$ or $\left\lfloor\sqrt{\frac{f(u)^{2}+f(v)^{2}}{2}}\right\rfloor$, then the edge labels are distinct and are from $\{1,2, \cdots, q\}$. In this case $f$ is called root square mean labeling of $G$.

Definition 1.2: A walk in which $u_{1} u_{2} \cdots u_{n}$ are distinct is called a path. A path on $n$ vertices is denoted by $P_{n}$.

Definition 1.3: A closed path is called a cycle. A cycle on $n$ vertices is denoted by $C_{n}$.

Definition 1.4: Let $G=(V, E)$ be a graph with $V=S_{1} \cup S_{2} \cup \cdots \cup S_{t} \cup T$, where each $S_{i}$ is a set of vertices having at least two vertices and having the same degree and $T=V-\bigcup S_{i}$. The degree splitting graph of $G$ is denoted by $D S(G)$ and is obtained from $G$ by adding the vertices $w_{1}, w_{2}, \cdots, w_{t}$ and joining $w_{i}$ to each vertex of $S_{i}, 1 \leq i \leq t$. The graph $G$ and its degree splitting graph $D S(G)$ are given in Figure 1 .

Definition 1.5: The union of two graphs $G_{1}=\left(V_{1}, E_{1}\right)$ and $G_{2}=\left(V_{2}, E_{2}\right)$ is a graph $G=G_{1} \cup G_{2}$ with vertex set $V=V_{1} \cup V_{2}$ and the edge set $E=E_{1} \cup E_{2}$.

Theorem 1.6: Any path is a root square mean graph.

Theorem 1.7: Any cycle is a root square mean graph.

\section{Main Results}

Theorem 2.1: $n D S\left(P_{3}\right)$ is a root square mean graph.

Proof: The graph $\mathrm{DS}\left(P_{3}\right)$ is shown in Figure 2.

Let $G=n D S\left(P_{3}\right)$. Let the vertex set of $G$ be $V=V_{1} \cup V_{2} \cup \cdots \cup V_{n}$ where $V_{i}=\left\{v_{1}^{i}, v_{2}^{i}, v_{3}^{i}, w_{i}, 1 \leq i \leq n\right\}$. Define a function $f: V(G) \rightarrow\{1,2, \cdots, q+1\}$ by

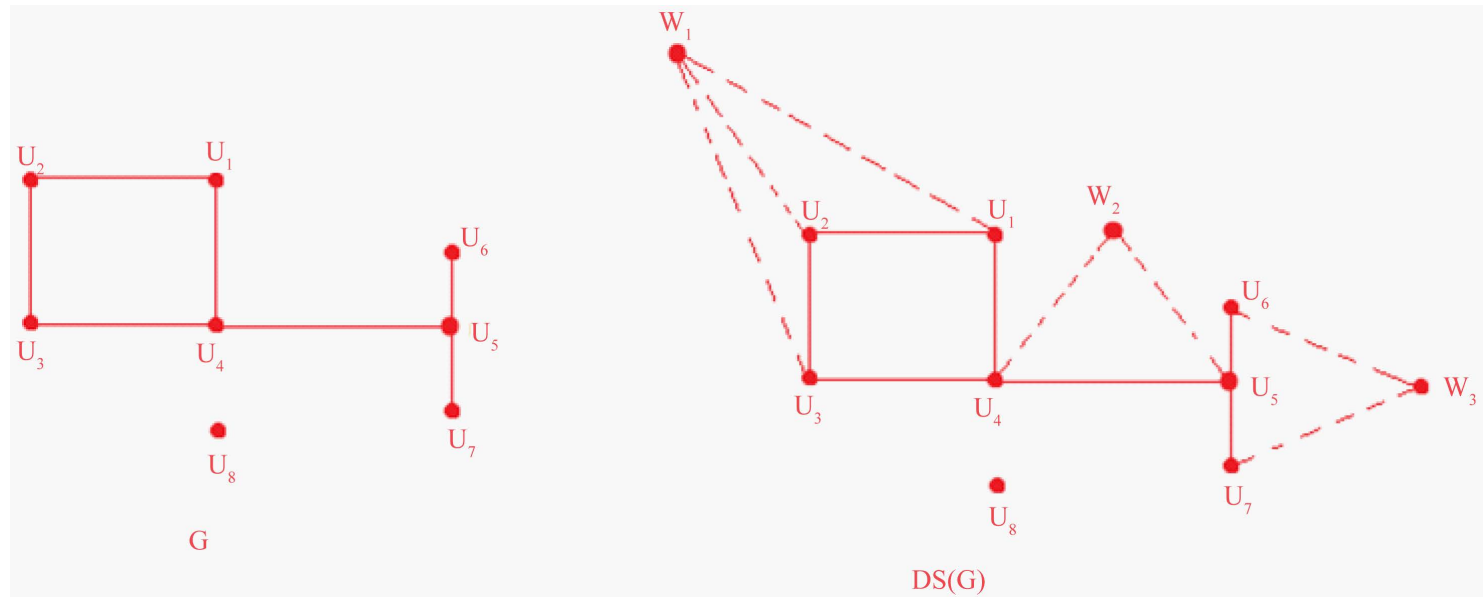

Figure 1. The graph $G$ and its degree splitting graph $D S(G)$.

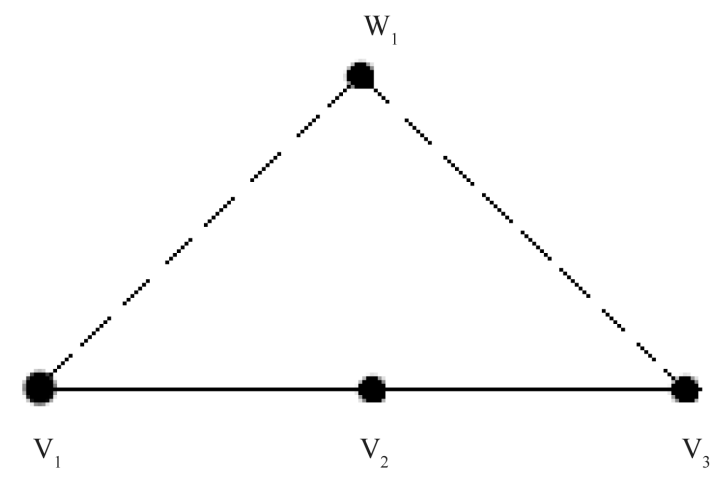

Figure 2. The graph $D S\left(P_{3}\right)$. 


$$
\begin{aligned}
& f\left(v_{1}^{i}\right)=4 i-3,1 \leq i \leq n \\
& f\left(v_{2}^{i}\right)=4 i-2,1 \leq i \leq n \\
& f\left(v_{3}^{i}\right)=4 i-1,1 \leq i \leq n \\
& f\left(w_{i}\right)=4 i, 1 \leq i \leq n
\end{aligned}
$$

Then the edges are labeled as

$$
\begin{aligned}
& f\left(v_{1}^{i} v_{2}^{i}\right)=4 i-3,1 \leq i \leq n-1 \\
& f\left(v_{2}^{i} v_{3}^{i}\right)=4 i-1,1 \leq i \leq n-1 \\
& f\left(v_{1}^{i} w_{i}\right)=4 i-2,1 \leq i \leq n-2 \\
& f\left(v_{3}^{i} w_{i}\right)=4 i, 1 \leq i \leq n-2
\end{aligned}
$$

Then the edge labels are distinct and are from $\{1,2, \cdots, q\}$. Hence by definition $1.1, G$ is a root square mean graph.

Example 2.2: Root square mean labeling of $4 D S\left(P_{3}\right)$ is shown in Figure 3.

Theorem 2.3: $4 D S\left(P_{4}\right)$ is a root square mean graph.

Proof: The graph $D S\left(P_{4}\right)$ is shown in Figure 4.

Let $G=n D S\left(P_{3}\right)$. Let the vertex set of $G$ be $V=V_{1} \cup V_{2} \cup \cdots \cup V_{n}$ where $V_{i}=\left\{v_{1}^{i}, v_{2}^{i}, v_{3}^{i}, v_{4}^{i}, w_{1}^{i}, w_{2}^{i}, 1 \leq i \leq n\right\}$. Define a function $f: V(G) \rightarrow\{1,2, \cdots, q+1\}$ by
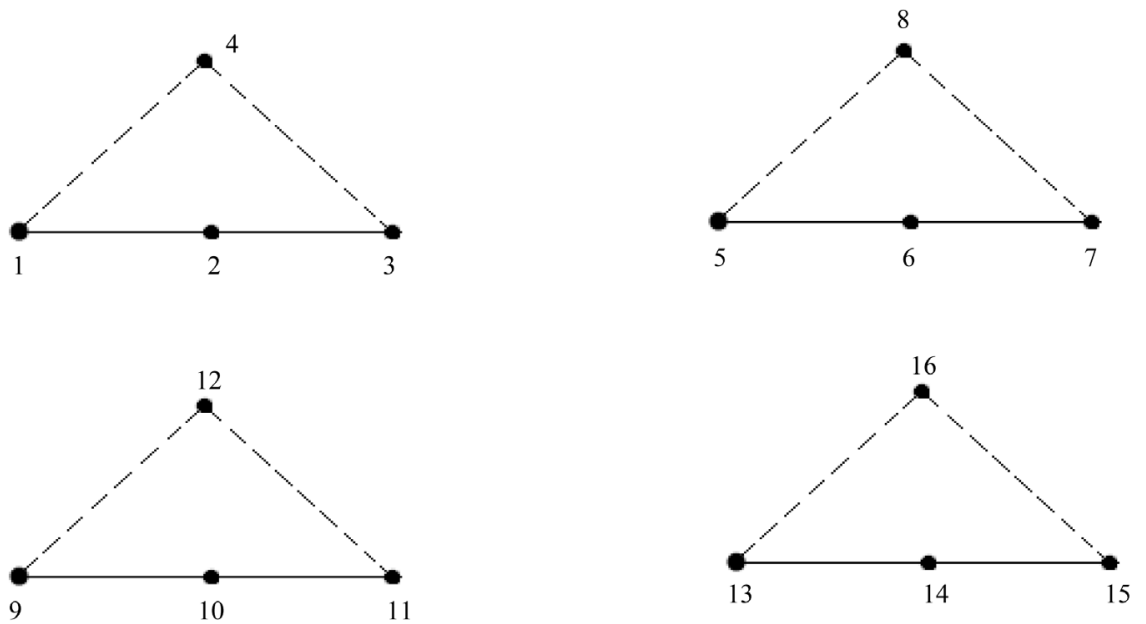

Figure 3. Root square mean labeling of $4 D S\left(P_{3}\right)$.

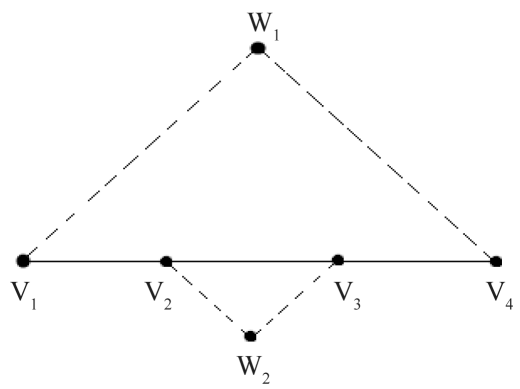

Figure 4. The graph $D S\left(P_{4}\right)$. 


$$
\begin{aligned}
& f\left(v_{1}^{i}\right)=7 i-5,1 \leq i \leq n \\
& f\left(v_{2}^{i}\right)=7 i-3,1 \leq i \leq n \\
& f\left(v_{3}^{i}\right)=7 i-1,1 \leq i \leq n \\
& f\left(v_{4}^{i}\right)=7 i-4,1 \leq i \leq n \\
& f\left(w_{1}^{i}\right)=7 i-6,1 \leq i \leq n \\
& f\left(w_{2}^{i}\right)=7 i, 1 \leq i \leq n
\end{aligned}
$$

Then the edges are labeled as

$$
\begin{aligned}
& f\left(v_{1}^{i} v_{2}^{i}\right)=7 i-4,1 \leq i \leq n \\
& f\left(v_{2}^{i} v_{3}^{i}\right)=7 i-2,1 \leq i \leq n \\
& f\left(v_{3}^{i} v_{4}^{i}\right)=7 i-3,1 \leq i \leq n \\
& f\left(v_{1}^{i} w_{1}^{i}\right)=7 i-6,1 \leq i \leq n \\
& f\left(w_{1}^{i} v_{4}^{i}\right)=7 i-5,1 \leq i \leq n \\
& f\left(v_{2}^{i} w_{2}^{i}\right)=7 i-1,1 \leq i \leq n \\
& f\left(v_{3}^{i} w_{2}^{i}\right)=7 i, 1 \leq i \leq n
\end{aligned}
$$

Then the edge labels are distinct and are from $\{1,2, \cdots, q\}$. Hence by definition $1.1, G$ is a root square mean graph.

Example 2.4: Root square mean labeling of $4 D S\left(P_{3}\right)$ is shown in Figure 5.

Theorem 2.5: $n D S\left(P_{2} \odot K_{1}\right)$ is a root square mean graph.

Proof: The graph $D S\left(P_{2} \odot K_{1}\right)$ is shown in Figure 6.

Let $G=n D S\left(P_{2} \odot K_{1}\right)$. Let the vertex set of $G$ be $V=V_{1} \cup V_{2} \cup \cdots \cup V_{n}$ where $V_{i}=\left\{v_{1}^{i}, v_{2}^{i}, v_{3}^{i}, v_{4}^{i}, w_{1}^{i}, w_{2}^{i}, 1 \leq i \leq n\right\}$. Define a function $f: V(G) \rightarrow\{1,2, \cdots, q+1\}$ by
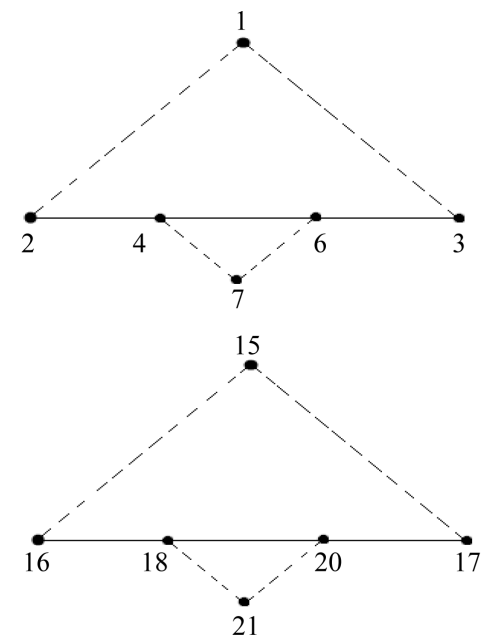
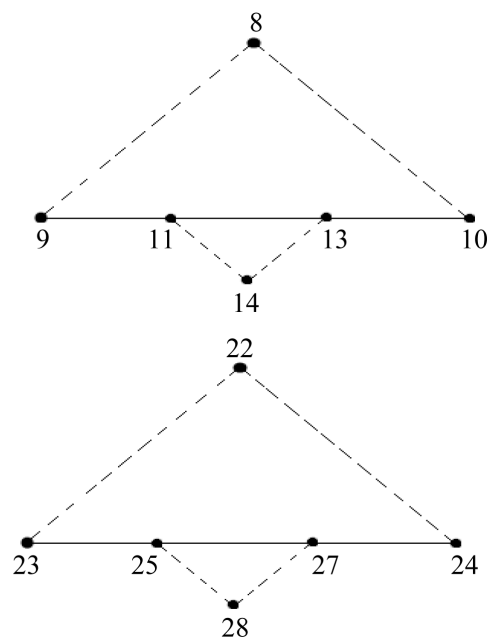

Figure 5. Root square mean labeling of $4 D S\left(P_{3}\right)$. 


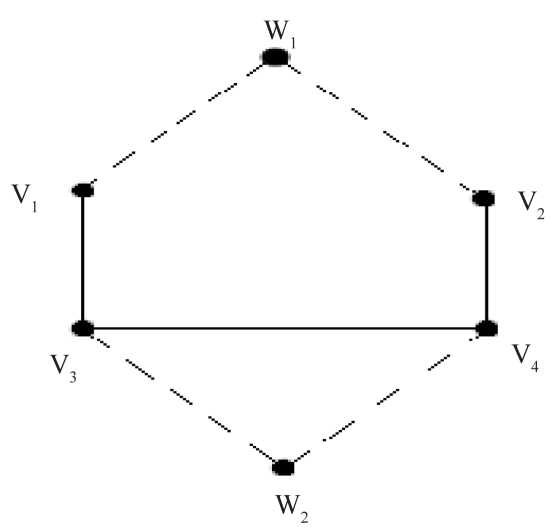

Figure 6. The graph $D S\left(P_{2} \odot K_{1}\right)$.

$$
\begin{aligned}
& f\left(v_{1}^{i}\right)=7 i-5,1 \leq i \leq n \\
& f\left(v_{2}^{i}\right)=7 i-4,1 \leq i \leq n \\
& f\left(v_{3}^{i}\right)=7 i-2,1 \leq i \leq n \\
& f\left(v_{4}^{i}\right)=7 i-1,1 \leq i \leq n \\
& f\left(w_{1}^{i}\right)=7 i-6,1 \leq i \leq n \\
& f\left(w_{2}^{i}\right)=7 i, 1 \leq i \leq n
\end{aligned}
$$

Then the edges are labeled as

$$
\begin{aligned}
& f\left(v_{1}^{i} v_{3}^{i}\right)=7 i-4,1 \leq i \leq n \\
& f\left(v_{3}^{i} v_{4}^{i}\right)=7 i-2,1 \leq i \leq n \\
& f\left(v_{4}^{i} v_{2}^{i}\right)=7 i-3,1 \leq i \leq n \\
& f\left(v_{1}^{i} w_{1}^{i}\right)=7 i-6,1 \leq i \leq n \\
& f\left(v_{2}^{i} w_{1}^{i}\right)=7 i-5,1 \leq i \leq n \\
& f\left(v_{3}^{i} w_{2}^{i}\right)=7 i-1,1 \leq i \leq n \\
& f\left(v_{4}^{i} w_{2}^{i}\right)=7 i, 1 \leq i \leq n
\end{aligned}
$$

Then the edge labels are distinct and are from $\{1,2, \cdots, q\}$. Hence by definition $1.1, G$ is a root square mean graph.

Example 2.6: The labeling pattern of $4 D S\left(P_{2} \odot K_{1}\right)$ is shown in Figure 7.

Theorem 2.7: $n D S\left(P_{2} \odot K_{2}\right)$ is a root square mean graph.

Proof: The graph $D S\left(P_{2} \odot K_{2}\right)$ is shown in Figure 8.

Let $G=n D S\left(P_{2} \odot K_{2}\right)$. Let the vertex set of $G$ be $V=V_{1} \cup V_{2} \cup \cdots \cup V_{n}$ where $V_{i}=\left\{v_{1}^{i}, v_{2}^{i}, v_{3}^{i}, v_{4}^{i}, v_{5}^{i}, v_{6}^{i}, w_{1}^{i}, w_{2}^{i}, 1 \leq i \leq n\right\}$. Define a function $f: V(G) \rightarrow\{1,2, \cdots, q+1\}$ by

$$
\begin{aligned}
& f\left(v_{1}^{i}\right)=11 i-5,1 \leq i \leq n \\
& f\left(v_{2}^{i}\right)=11 i-3,1 \leq i \leq n
\end{aligned}
$$



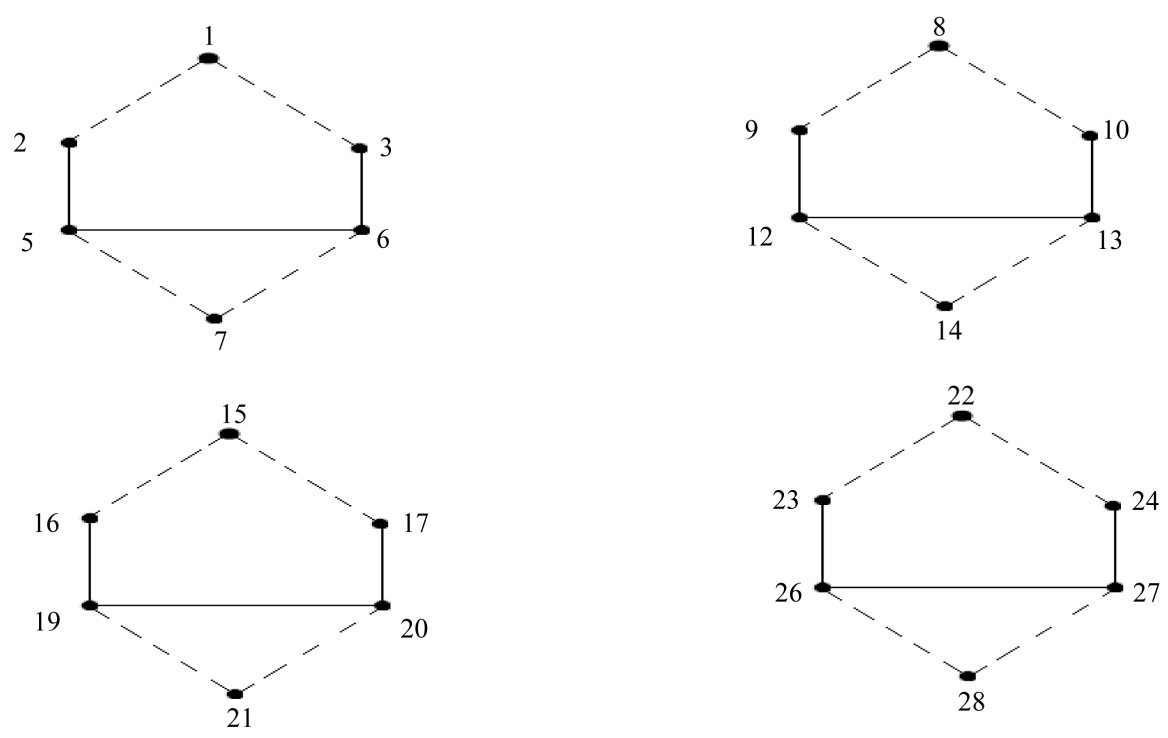

Figure 7. The labeling pattern of $4 D S\left(P_{2} \odot K_{1}\right)$.

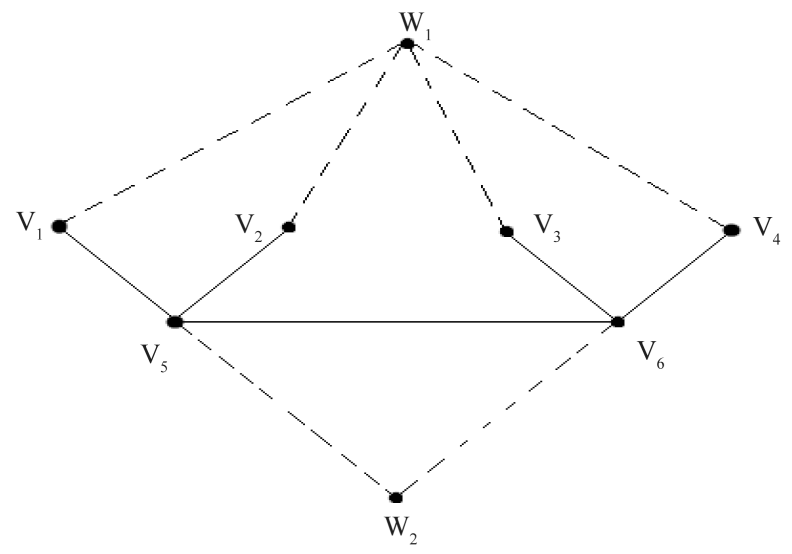

Figure 8. The graph $D S\left(P_{2} \odot K_{2}\right)$.

$$
\begin{aligned}
& f\left(v_{3}^{i}\right)=11 i-2,1 \leq i \leq n \\
& f\left(v_{4}^{i}\right)=11 i-1,1 \leq i \leq n \\
& f\left(v_{5}^{i}\right)=11 i-9,1 \leq i \leq n \\
& f\left(v_{6}^{i}\right)=11 i-7,1 \leq i \leq n \\
& f\left(w_{1}^{i}\right)=11 i, 1 \leq i \leq n \\
& f\left(w_{2}^{i}\right)=11 i-10,1 \leq i \leq n
\end{aligned}
$$

Then the edges are labeled as

$$
\begin{aligned}
& f\left(v_{5}^{i} v_{6}^{i}\right)=11 i-8,1 \leq i \leq n \\
& f\left(v_{5}^{i} v_{1}^{i}\right)=11 i-7,1 \leq i \leq n
\end{aligned}
$$




$$
\begin{aligned}
& f\left(v_{5}^{i} v_{2}^{i}\right)=11 i-6,1 \leq i \leq n \\
& f\left(v_{6}^{i} v_{3}^{i}\right)=11 i-5,1 \leq i \leq n \\
& f\left(v_{6}^{i} v_{4}^{i}\right)=11 i-4,1 \leq i \leq n \\
& f\left(v_{1}^{i} w_{1}^{i}\right)=11 i-3,1 \leq i \leq n \\
& f\left(v_{2}^{i} w_{1}^{i}\right)=11 i-2,1 \leq i \leq n \\
& f\left(v_{3}^{i} w_{1}^{i}\right)=11 i-1,1 \leq i \leq n \\
& f\left(v_{4}^{i} w_{1}^{i}\right)=11 i, 1 \leq i \leq n \\
& f\left(v_{5}^{i} w_{2}^{i}\right)=11 i-10,1 \leq i \leq n \\
& f\left(v_{6}^{i} w_{2}^{i}\right)=11 i-9,1 \leq i \leq n
\end{aligned}
$$

Then the edge labels are distinct and are from $\{1,2, \cdots, q\}$. Hence by definition $1.1, G$ is a root square mean graph.

Example 2.8: The labeling pattern of $2 D S\left(P_{2} \odot K_{2}\right)$ is shown in Figure 9.

Theorem 2.9: $n D S\left(P_{2} \odot \overline{K_{3}}\right)$ is a root square mean graph.

Proof: The graph $D S\left(P_{2} \odot \overline{K_{3}}\right)$ is shown in Figure 10.

Let $G=n D S\left(P_{2} \odot \overline{K_{3}}\right)$. Let the vertex set of $G$ be $V=V_{1} \cup V_{2} \cup \cdots \cup V_{n}$ where $V_{i}=\left\{v_{1}^{i}, v_{2}^{i}, v_{3}^{i}, v_{4}^{i}, v_{5}^{i}, v_{6}^{i}, v_{7}^{i}, v_{8}^{i}, w_{1}^{i}, w_{2}^{i}, 1 \leq i \leq n\right\}$.

Define a function $f: V(G) \rightarrow\{1,2, \cdots, q+1\}$ by

$$
\begin{aligned}
& f\left(v_{1}^{i}\right)=15 i-11,1 \leq i \leq n \\
& f\left(v_{2}^{i}\right)=15 i-8,1 \leq i \leq n \\
& f\left(v_{3}^{i}\right)=15 i-6,1 \leq i \leq n \\
& f\left(v_{4}^{i}\right)=15 i-5,1 \leq i \leq n \\
& f\left(v_{5}^{i}\right)=15 i-3,1 \leq i \leq n \\
& f\left(v_{6}^{i}\right)=15 i-2,1 \leq i \leq n \\
& f\left(w_{1}^{i}\right)=15 i, 1 \leq i \leq n \\
& f\left(w_{2}^{i}\right)=15 i-14,1 \leq i \leq n \\
& f\left(v_{7}^{i}\right)=15 i-13,1 \leq i \leq n \\
& f\left(v_{8}^{i}\right)=15 i-12,1 \leq i \leq n
\end{aligned}
$$

Then the edges are labeled as

$$
\begin{aligned}
& f\left(v_{7}^{i} v_{1}^{i}\right)=15 i-11,1 \leq i \leq n \\
& f\left(v_{7}^{i} v_{2}^{i}\right)=15 i-10,1 \leq i \leq n \\
& f\left(v_{7}^{i} v_{3}^{i}\right)=15 i-9,1 \leq i \leq n
\end{aligned}
$$



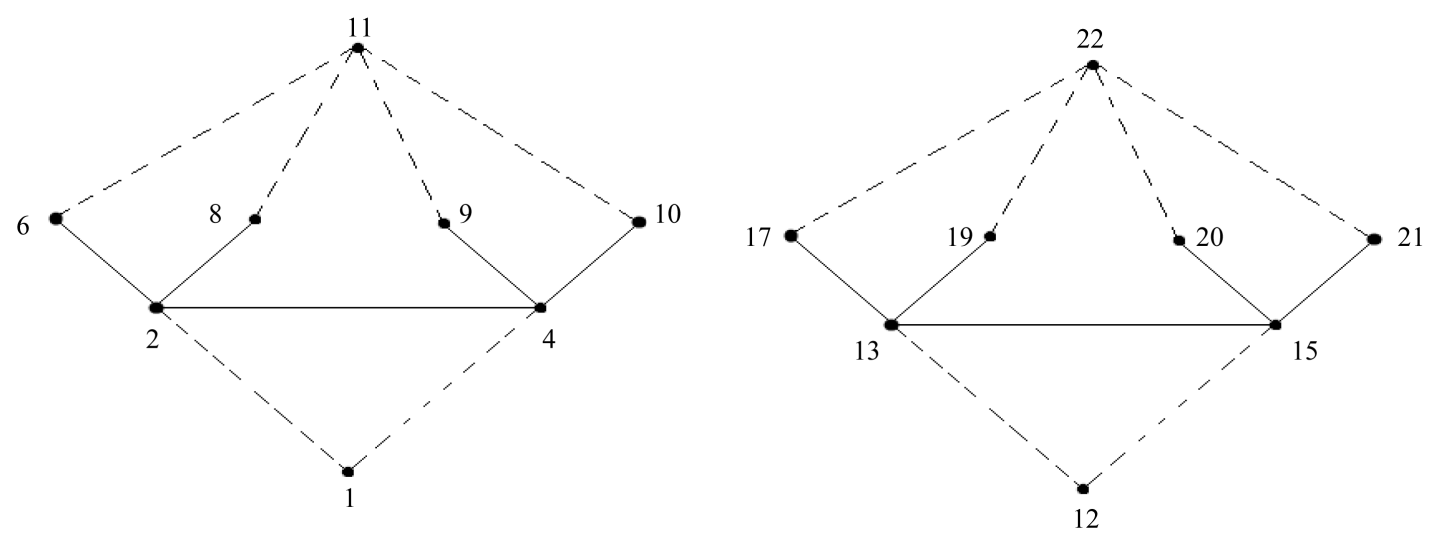

Figure 9. The labeling pattern of $2 D S\left(P_{2} \odot K_{2}\right)$.

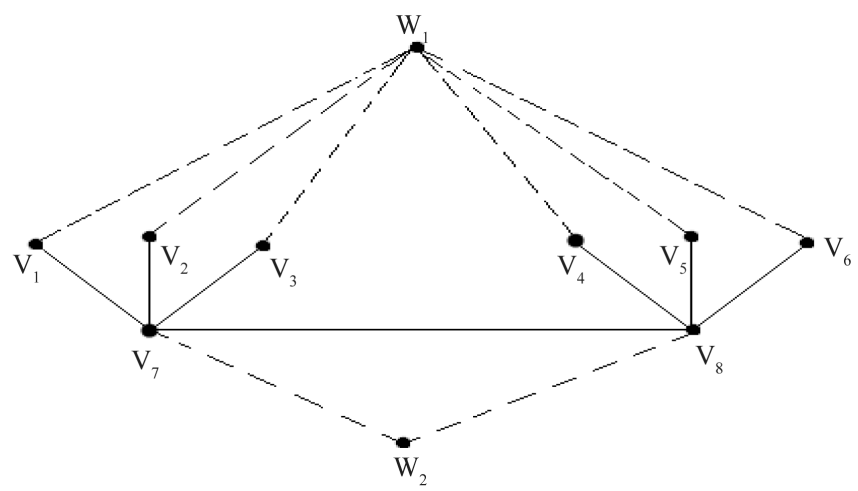

Figure 10. The graph $D S\left(P_{2} \odot \overline{K_{3}}\right)$.

$$
\begin{aligned}
& f\left(v_{8}^{i} v_{4}^{i}\right)=15 i-8,1 \leq i \leq n \\
& f\left(v_{8}^{i} v_{5}^{i}\right)=15 i-7,1 \leq i \leq n \\
& f\left(v_{8}^{i} v_{6}^{i}\right)=15 i-6,1 \leq i \leq n \\
& f\left(v_{7}^{i} w_{2}^{i}\right)=15 i-14,1 \leq i \leq n \\
& f\left(v_{8}^{i} w_{2}^{i}\right)=15 i-13,1 \leq i \leq n \\
& f\left(v_{1}^{i} w_{1}^{i}\right)=15 i-5,1 \leq i \leq n \\
& f\left(v_{2}^{i} w_{1}^{i}\right)=15 i-4,1 \leq i \leq n \\
& f\left(v_{3}^{i} w_{1}^{i}\right)=15 i-3,1 \leq i \leq n \\
& f\left(v_{4}^{i} w_{1}^{i}\right)=15 i-2,1 \leq i \leq n \\
& f\left(v_{5}^{i} w_{1}^{i}\right)=15 i-1,1 \leq i \leq n \\
& f\left(v_{6}^{i} w_{1}^{i}\right)=15 i, 1 \leq i \leq n \\
& f\left(v_{7}^{i} v_{8}^{i}\right)=15 i-12,1 \leq i \leq n
\end{aligned}
$$


Then the edge labels are distinct and are from $\{1,2, \cdots, q\}$. Hence by definition $1.1, G$ is a root square mean graph.

Example 2.10: The root square mean labeling of $2 D S\left(P_{2} \odot \overline{K_{3}}\right)$ is shown in Figure 11.

Theorem 2.11: $n D S\left(P_{3} \odot K_{1}\right)$ is a root square mean graph.

Proof: The graph $D S\left(P_{3} \odot K_{1}\right)$ is shown in Figure 12 .

Let $G=n D S\left(P_{3} \odot K_{1}\right)$. Let its vertex set be $V=V_{1} \cup V_{2} \cup \cdots \cup V_{n}$ where $V_{i}=\left\{v_{1}^{i}, v_{2}^{i}, v_{3}^{i}, v_{4}^{i}, v_{5}^{i}, v_{6}^{i}, w_{1}^{i}, w_{2}^{i}, 1 \leq i \leq n\right\}$.

Define a function $f: V(G) \rightarrow\{1,2, \cdots, q+1\}$ by

$$
\begin{aligned}
& f\left(v_{1}^{i}\right)=10 i-3,1 \leq i \leq n \\
& f\left(v_{2}^{i}\right)=10 i-2,1 \leq i \leq n \\
& f\left(v_{3}^{i}\right)=10 i-1,1 \leq i \leq n \\
& f\left(v_{4}^{i}\right)=10 i-8,1 \leq i \leq n \\
& f\left(v_{5}^{i}\right)=10 i-5,1 \leq i \leq n \\
& f\left(v_{6}^{i}\right)=10 i-6,1 \leq i \leq n \\
& f\left(w_{1}^{i}\right)=10 i, 1 \leq i \leq n \\
& f\left(w_{2}^{i}\right)=10 i-9,1 \leq i \leq n
\end{aligned}
$$
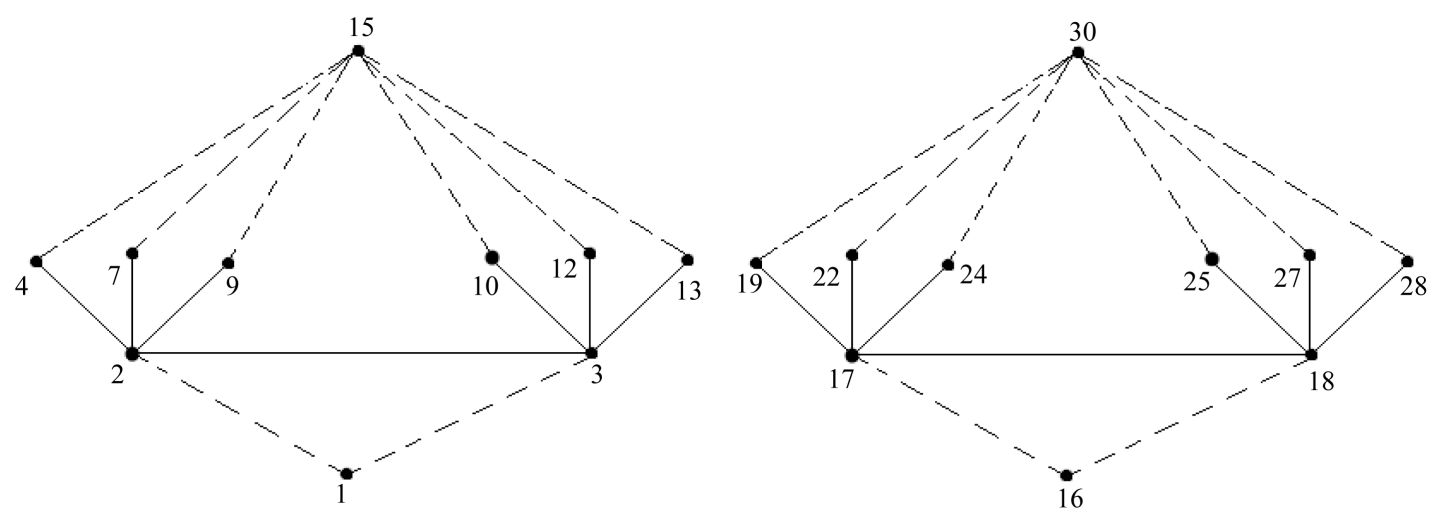

Figure 11. The root square mean labeling of $2 D S\left(P_{2} \odot \overline{K_{3}}\right)$.

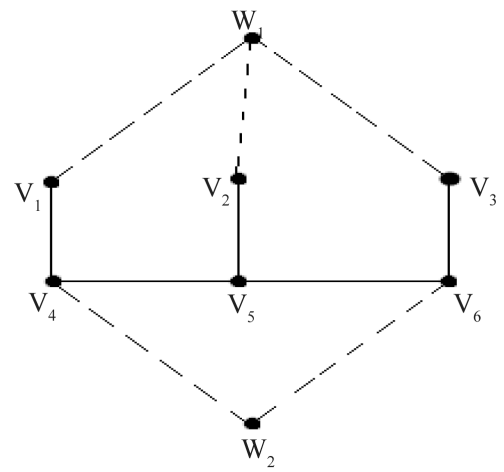

Figure 12. The graph $D S\left(P_{3} \odot K_{1}\right)$. 
Then the edges are labeled as

$$
\begin{aligned}
& f\left(v_{4}^{i} v_{1}^{i}\right)=10 i-5,1 \leq i \leq n \\
& f\left(v_{5}^{i} v_{2}^{i}\right)=10 i-4,1 \leq i \leq n \\
& f\left(v_{6}^{i} v_{3}^{i}\right)=10 i-3,1 \leq i \leq n \\
& f\left(v_{1}^{i} w_{1}^{i}\right)=10 i-2,1 \leq i \leq n \\
& f\left(v_{2}^{i} w_{1}^{i}\right)=10 i-1,1 \leq i \leq n \\
& f\left(v_{3}^{i} w_{1}^{i}\right)=10 i, 1 \leq i \leq n \\
& f\left(v_{4}^{i} w_{2}^{i}\right)=10 i-9,1 \leq i \leq n \\
& f\left(v_{6}^{i} w_{2}^{i}\right)=10 i-8,1 \leq i \leq n
\end{aligned}
$$

Then the edge labels are distinct and are from $\{1,2, \cdots, q\}$. Hence by definition $1.1, G$ is a root square mean graph.

Example 2.12: The labeling pattern of $3 D S\left(P_{3} \odot K_{1}\right)$ is shown in Figure 13.

Theorem 2.13: $n D S\left(K_{1,3}\right)$ is a root square mean graph.

Proof: The graph $D S\left(K_{1,3}\right)$ is shown in Figure 14.

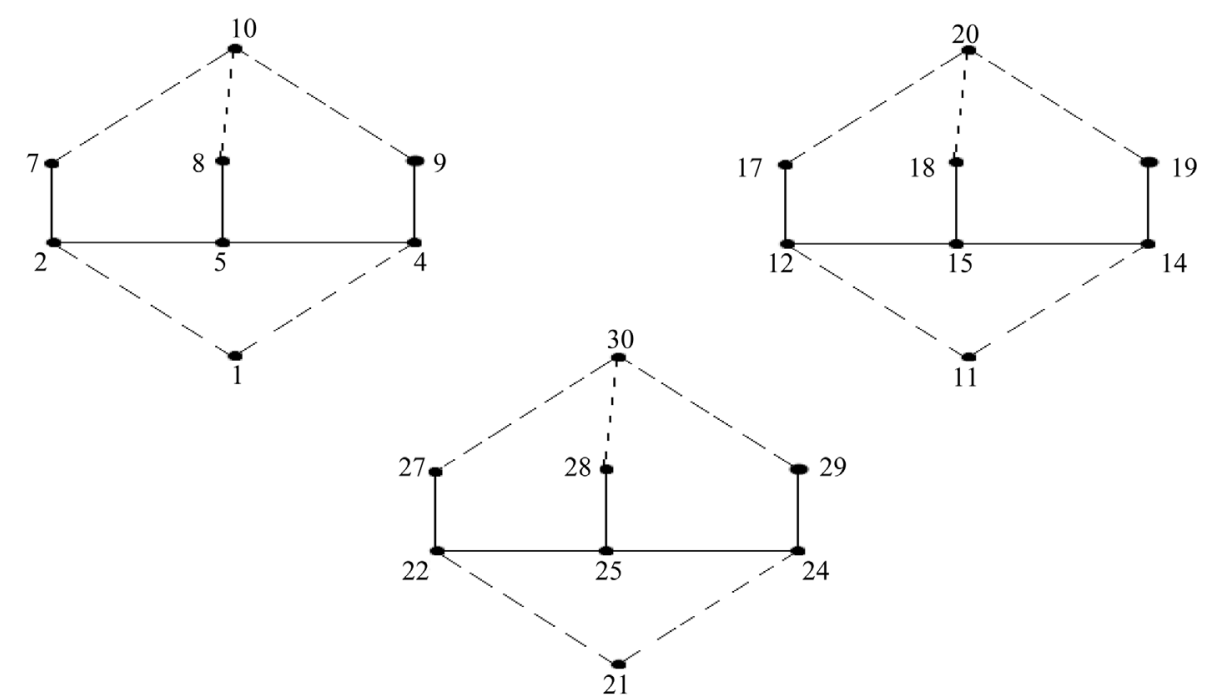

Figure 13. The labeling pattern of $3 D S\left(P_{3} \odot K_{1}\right)$.

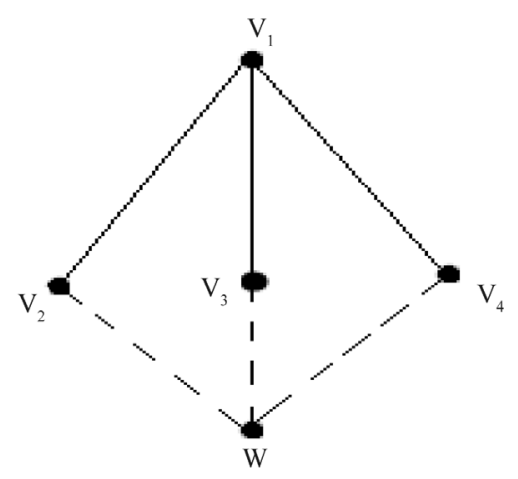

Figure 14. The graph $D S\left(K_{1,3}\right)$. 
Let $G=n D S\left(K_{1,3}\right)$. Let its vertex set be $V=V_{1} \cup V_{2} \cup \cdots \cup V_{n}$ where $V_{i}=\left\{v_{1}^{i}, v_{2}^{i}, v_{3}^{i}, v_{4}^{i}, w^{i}, 1 \leq i \leq n\right\}$.

Define a function $f: V(G) \rightarrow\{1,2, \cdots, q+1\}$ by

$$
\begin{aligned}
& f\left(v_{1}^{i}\right)=6 i-5,1 \leq i \leq n \\
& f\left(v_{2}^{i}\right)=6 i-4,1 \leq i \leq n \\
& f\left(v_{3}^{i}\right)=6 i-2,1 \leq i \leq n \\
& f\left(v_{4}^{i}\right)=6 i-1,1 \leq i \leq n \\
& f\left(w^{i}\right)=6 i, 1 \leq i \leq n
\end{aligned}
$$

Then the edges are labeled as

$$
\begin{aligned}
& f\left(v_{1}^{i} v_{2}^{i}\right)=6 i-5,1 \leq i \leq n \\
& f\left(v_{1}^{i} v_{3}^{i}\right)=6 i-4,1 \leq i \leq n \\
& f\left(v_{1}^{i} v_{4}^{i}\right)=6 i-3,1 \leq i \leq n \\
& f\left(v_{2}^{i} w^{i}\right)=6 i-2,1 \leq i \leq n \\
& f\left(v_{3}^{i} w^{i}\right)=6 i-1,1 \leq i \leq n \\
& f\left(v_{4}^{i} w^{i}\right)=6 i, 1 \leq i \leq n
\end{aligned}
$$

Then the edge labels are distinct and are from $\{1,2, \cdots, q\}$. Hence by definition $1.1, G$ is a root square mean graph.

Example 2.14: The labeling pattern of $4 D S\left(K_{1,3}\right)$ is shown in Figure 15.

Theorem 2.15: $n D S\left(C_{3} \hat{O} K_{1,2}\right)$ is a root square mean graph.

Proof: The graph $D S\left(C_{3} \hat{O} K_{1,2}\right)$ is shown in Figure 16.

Let $G=n D S\left(C_{3} \hat{O} K_{1,2}\right)$. Let its vertex set be $V=V_{1} \cup V_{2} \cup \cdots \cup V_{n}$
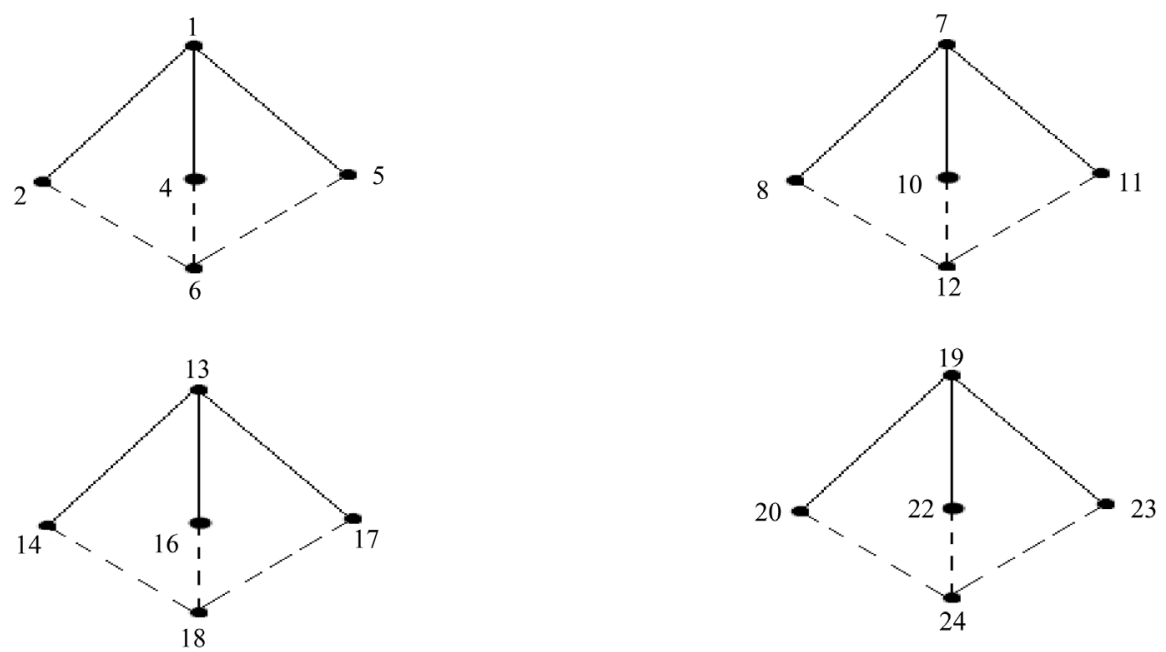

Figure 15. The labeling pattern of $4 D S\left(K_{1,3}\right)$. 


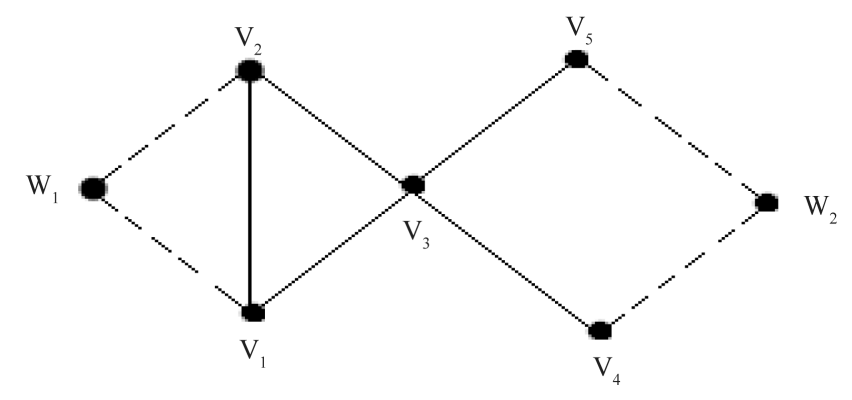

Figure 16. The graph $D S\left(C_{3} \hat{\mathrm{O}} K_{1,2}\right)$.

where $V_{i}=\left\{v_{1}^{i}, v_{2}^{i}, v_{3}^{i}, v_{4}^{i}, v_{5}^{i}, w_{1}^{i}, w_{2}^{i}, 1 \leq i \leq n\right\}$.

Define a function $f: V(G) \rightarrow\{1,2, \cdots, q+1\}$ by

$$
\begin{aligned}
& f\left(v_{1}^{i}\right)=9 i-7,1 \leq i \leq n \\
& f\left(v_{2}^{i}\right)=9 i-5,1 \leq i \leq n \\
& f\left(v_{3}^{i}\right)=9 i-4,1 \leq i \leq n \\
& f\left(v_{4}^{i}\right)=9 i-2,1 \leq i \leq n \\
& f\left(v_{5}^{i}\right)=9 i-1,1 \leq i \leq n \\
& f\left(w_{1}^{i}\right)=9 i-8,1 \leq i \leq n \\
& f\left(w_{2}^{i}\right)=9 i, 1 \leq i \leq n
\end{aligned}
$$

Then the edges are labeled as

$$
\begin{aligned}
& f\left(w_{1}^{i} v_{1}^{i}\right)=9 i-8,1 \leq i \leq n \\
& f\left(w_{1}^{i} v_{2}^{i}\right)=9 i-7,1 \leq i \leq n \\
& f\left(v_{1}^{i} v_{2}^{i}\right)=9 i-6,1 \leq i \leq n \\
& f\left(v_{1}^{i} v_{3}^{i}\right)=9 i-5,1 \leq i \leq n \\
& f\left(v_{2}^{i} v_{3}^{i}\right)=9 i-4,1 \leq i \leq n \\
& f\left(v_{3}^{i} v_{4}^{i}\right)=9 i-3,1 \leq i \leq n \\
& f\left(v_{3}^{i} v_{5}^{i}\right)=9 i-2,1 \leq i \leq n \\
& f\left(v_{4}^{i} w_{2}^{i}\right)=9 i-1,1 \leq i \leq n \\
& f\left(v_{5}^{i} w_{2}^{i}\right)=9 i, 1 \leq i \leq n
\end{aligned}
$$

Then the edge labels are distinct and are from $\{1,2, \cdots, q\}$. Hence by definition $1.1, G$ is a root square mean graph. 

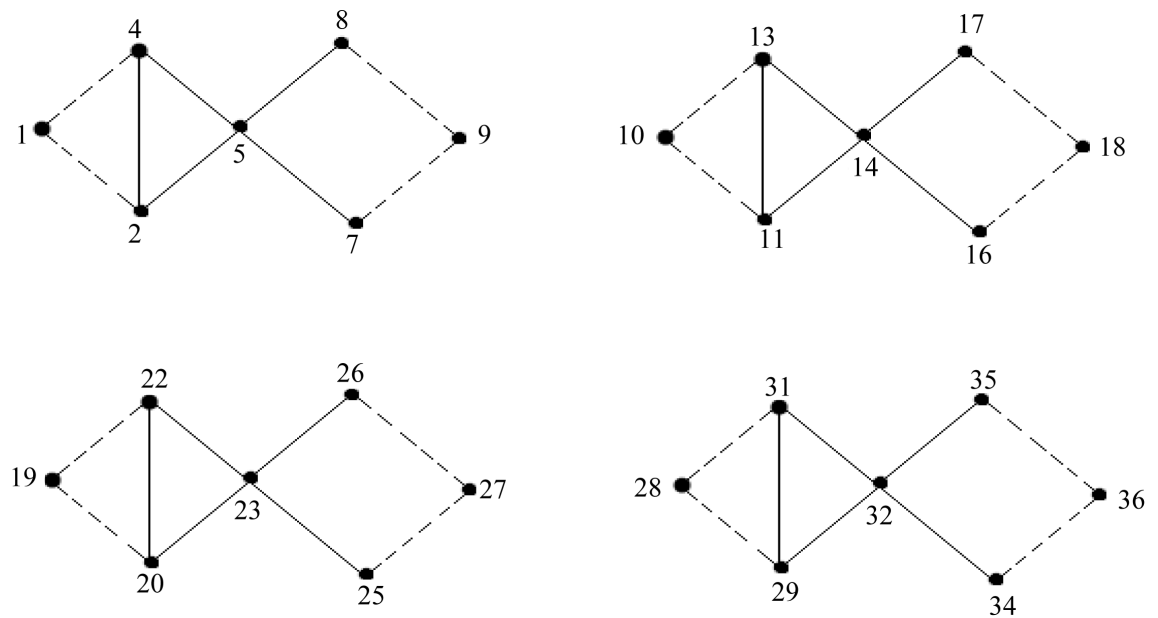

Figure 17. The root square mean labeling of $4 D S\left(C_{3} \hat{O} K_{1,2}\right)$.

Example 2.16: The root square mean labeling of $4 D S\left(C_{3} \hat{\mathrm{O}} K_{1,2}\right)$ is given in Figure 17.

\section{References}

[1] Gallian, J.A. (2012) A Dynamic Survey of Graph Labeling. The Electronic Journal of Combinatories.

[2] Harary, F. (1988) Graph Theory. Narosa Publishing House Reading, New Delhi.

[3] Sandhya, S.S., Jayasekaran, C. and Raj, C.D. (2013) Harmonic Mean Labeling of Degree Splitting Graphs. Bulletin of Pure and Applied Sciences, 32E, 99-112.

[4] Sandhya, S.S., Somasundaram, S. and Anusa, S. (2014) Root Square Mean Labeling of Graphs. International Journal of Contemporary Mathematical Sciences, 9, 667-676.

[5] Sandhya, S.S., Somasundaram, S. and Anusa, S. (2015) Some More Results on Root Square Mean Graphs. Journal of Mathematics Research, 7.

[6] Sandhya, S.S., Somasundaram, S. and Anusa, S. (2014) Root Square Mean Labeling of Some New Disconnected Graphs. International Journal of Mathematics Trends and Technology, 15, 85-92. http://dx.doi.org/10.14445/22315373/IJMTT-V15P511

[7] Sandhya, S.S., Somasundaram, S. and Anusa, S. (2014) Root Square Mean Labeling of Subdivision of Some More Graphs. International Journal of Mathematics Research, 6, 253-266.

[8] Sandhya, S.S., Somasundaram, S. and Anusa, S. (2014) Some New Results on Root Square Mean Labeling. International Journal of Mathematical Archive, 5, 130-135.

[9] Sandhya, S.S., Somasundaram, S. and Anusa, S. (2015) Root Square Mean Labeling of Subdivision of Some Graphs. Global Journal of Theoretical and Applied Mathematics Sciences, 5, 1-11.

[10] Sandhya, S.S., Somasundaram, S. and Anusa, S. (2015) Root Square Mean Labeling of Some More Disconnected Graphs. International Mathematical Forum, 10, 25-34.

[11] Sandhya, S.S., Somasundaram, S. and Anusa, S. (2015) Some Results on Root Square Mean Graphs. Communicated to Journal of Scientific Research. 